\title{
Association of CCR5 $\Delta 32$ deletion with early death in multiple sclerosis
}

Radhika Gade-Andavolu, $P h D^{1}$, David E. Comings, $M D^{2}$, James MacMurray, PhD' ${ }^{2}$, Masoud Rostamkhani, $M S^{2}$, Li S.- C. Cheng PhD ${ }^{3}$, Wallace W. Tourtellotte, $M D, P h D^{4}$, and Lawrence A. Cone, $M D, D S c^{1,5}$

\begin{abstract}
Purpose: The interaction between chemokines and their receptors is extremely important in controlling $\mathrm{T}$ cell migration into sites of CNS inflammation. Because trafficking of inflammatory $T$ cells into the central nervous system (CNS) is a key player in the pathogenesis of multiple sclerosis (MS), we investigated the possible association of CCR5 $\Delta 32$ deletion in this disorder. Methods: DNA isolated from postmortem brain tissue samples of 132 patients with MS and from blood tissue samples of 163 gender and ethnicity-matched healthy controls was used to screen for the CCR5 $\Delta 32$ deletion allele. Results: An increased frequency of 32-bp deletion allele was found to be associated with early death $(P=0.00005)$ and with a progressive reduction in the years of survival (onset to death). The death hazard ratio of CCR5 with deletion versus no deletion was 2.12, suggesting that MS patients with the 32-bp deletion have twice the mortality rate of patients with the normal genotype. This effect was more significant in females (hazard ratio 3.58). Conclusion: A strong association of the CCR5 32 deletion with early death could serve as a prognostic marker for MS. Genet Med 2004:6(3):126-131.
\end{abstract}

Key Words: multiple sclerosis, polymerase chain reaction, chemokine receptor, experimental autoimmune encephalomyelitis

Multiple sclerosis (MS) is a chronic demyelinating disorder pathologically characterized by an infiltration of monocytes and T-lymphocytes into the brain parenchyma, destruction of oligodendrocytes, and the loss of myelin. The role of chemokines and chemokine receptors is particularly important in MS, where myelin-destructive inflammation occurs inside the blood-brain barrier and is related to the influx of peripheral proinflammatory $\mathrm{T}$ cells into the CNS. Chemokines play a significant role in the migration of monocytes and $\mathrm{T}$ cells and also have been implicated in the onset or progression of MS and experimental autoimmune encephalomyelitis (EAE). ${ }^{1,2}$

CCR5, a seven transmembrane spanning G protein-coupled receptor, is a specific binding site for the CC-chemokines and is expressed on T-helper cells. Several studies have ascribed to CCR5 surface expression levels an important role in HIV-1 entry and pathogenesis, ${ }^{3}$ and a CCR $5 \Delta 32$ mutation (homozygous deletion) almost invariably protects from HIV-1 infection. ${ }^{4,5}$ The heterozygotes demonstrate a delay in progression of the disease ${ }^{6-8}$ lower viral loads and higher $\mathrm{CD} 4^{+}$counts. $^{9}$

\footnotetext{
${ }^{1}$ Genetic Research Institute of the Desert, Eisenhower Medical Center, Rancho Mirage, California; ${ }^{2}$ Department of Medical Genetics, City of Hope Medical Center, Duarte, California; ${ }^{3}$ Department of Bio statistics, City of Hope Medical Center, Duarte, California; ${ }^{4}$ Neurology \& Research Services, VA, West Los Angeles Healthcare Center \& Department of Neurology, UCLA, Los Angeles, California; and ${ }^{5}$ Sections of Immunology and Infectious Diseases, Eisenhower Medical Center, Rancho Mirage, California.

Dr. Lawrence A. Cone, 39000 Bob Hope Drive, Eisenhower Medical Center, Probst \# 308, Genetic Research Institute of the Desert, Rancho Mirage, CA 92270.

Received: November 21, 2003.

Accepted: February 17, 2004.
}

DOI: 10.1097/01.GIM.0000127274.45301.54
Aberrant production of chemokines has been described in humans and experimental CNS demyelinating lesions. ${ }^{10,11}$ $\beta$-Chemokine receptors were examined in postmortem MS CNS tissue by immunohistochemistry, and an elevated expression of CCR2, CCR3, and CCR5 was noted. ${ }^{12-14}$ HHV6 virus was additionally found in the human brain specimens and a possible association with MS was suggested. ${ }^{15-17}$ Chemokine receptor expression studies showed CCR5 and CXCR3 to have annualized increase in T2 lesion loads, suggesting these chemokines play an important role in the development of new lesions in MS than in the long-term outcome of those lesions. ${ }^{18}$

The ligands for CCR5 include RANTES (regulated-uponactivation, normal $\mathrm{T}$ expressed and secreted), macrophage inflammatory protein- $1 \alpha$ (MIP- $1 \alpha$ ), and MIP- $1 \beta$. These ligands belong to the group of $\mathrm{CC}$ or $\beta$-chemokines and are found principally on Th-1 helper cells. ${ }^{19}$ Earlier studies in HIV infected individuals have shown that T-cells from CCR5 $\Delta 32$ heterozygotes express 5- to 10-fold less CCR5 than wild types after stimulation. In addition, $\mathrm{CD} 4^{+} \mathrm{T}$-cell clones from two homozygous persons for the CCR $5 \Delta 32$ mutation produced approximately 20 -fold more RANTES than wild-type clones. ${ }^{20}$ Analysis of cytokine and cytokine receptor gene expression in MS samples showed predominantly increased levels of several Th- 1 molecules (TGF-ss, RANTES, and MIP- $1 \alpha$ ), although some Th-2 genes ( $I L-3, I L-5$, and $I L-6 / I L-6 R)$ were found to be up regulated as well. ${ }^{21}$

Genetic studies indicate MS is likely to be a polygenic disorder, due to multiple gene associations. ${ }^{22-24}$ Some of the genes reported to be associated with MS include interleukin, ${ }^{25,26}$ CTLA4, ${ }^{27}$ HLA-DRB1 ${ }^{*} 1501$, and apolipoprotein E. ${ }^{28}$ Early- 
published reports ${ }^{29,30}$ of epidemiological studies of MS concluded that the disease was common in persons of Scandinavian descent: an ethnic group that exhibits a high prevalence of CCR5 $\Delta 32$ mutation. Among the European white population there is a north to south gradient of prevalence of the CCR5 $\Delta 32$ mutant allele, with the allelic frequencies highest in Scandinavia (16\%) and lowest in Sardinia (4\%), with a mean allelic frequency across the whole of Europe of $9.1 \%$, where as this variant was found in only $1 \%$ in individuals of African origin, $0 \%$ in Asians, and $9.8 \%$ in Caucasians. ${ }^{31,32}$

Regardless of the degenerative pathway, damaged or dead neurons are a hallmark of many diseases including Alzheimer's, Parkinson's, glaucoma, ischemia, and multiple sclerosis. Molecules such as cytokines, chemokines, reactive nitrogen/ oxygen species, and proteases play an important role in promoting and/or mediating neurodegeneration. ${ }^{33}$ Based upon the key role played by chemokines in the migration of macrophages and T cells in MS, and the importance of genetic factors associated and the possible viral or nonviral stimulation involved in the pathogenesis of the disease, we investigated the role of the naturally occurring 32 -bp deletion ( $\Delta 32$ allele) in the CCR5 gene in patients with MS.

\section{MATERIALS AND METHODS}

\section{Study material}

DNA was isolated from postmortem brain tissue taken from 132 MS cases of the Human Brain and Spinal Fluid Resource Center at the VA, Los Angeles Health Care Center, Los Angeles, CA. All cases were necropsy-confirmed based on white matter lesions and demyelination. The sample was composed of 47 male and 85 female, non-Hispanic Caucasians. The age (which was the age-at-death) distribution of MS cases had a range of $30-86$, with a mean age of 57.2; the age at onset of MS ranged from 18 to 57, with a mean onset age of 34.6. Chronic progressive (CP) and relapsing remitting (RR) MS subtyping was obtained by chart review for $83(\mathrm{CP}=38$; $\mathrm{RR}=45)$ of the subjects, whereas the remaining charts provided insufficient data for accurate sub typing. The control sample consisted of 163 adult college students from a nearby university ( 78 males and 85 females) from whom blood samples were obtained. All the healthy subjects included for comparison were non-Hispanic Caucasians, age range between 18 and 49, and a mean age of 34.3. Written informed consent was obtained from the control subjects, and the study was approved by the IRB at City of Hope Medical Center.

\section{Genetic analysis}

To detect CCR5 $\triangle 32$ mutation, genomic DNA was extracted from brain samples and whole blood by standard procedures. PCR-based assay was used to determine the presence of the CCR5 $\Delta 32$ deletion. The following oligo-nucleotides were designed to yield a 232-bp product for the wild type: forward, GRL12A* 5'-TGTTTGCGTCTCTCCCAG-3' and reverse, GRL12B $^{\star} 5^{\prime}-$ CACAGCCCTGTGCCTCTT-3'
PCR was performed using an initial denaturation step at $94^{\circ} \mathrm{C}$ for 4 minutes, then a second step with a denaturation $94^{\circ} \mathrm{C}$ for 45 seconds, annealing at $55^{\circ} \mathrm{C}$ for 45 seconds, and an elongation at $72^{\circ} \mathrm{C}$ for 45 seconds for 29 cycles with a final elongation step at $72^{\circ} \mathrm{C}$ for 6 minutes. PCR products were run on $12 \%$ polyacrylamide gels at constant $150 \mathrm{~V}$ for 2 hours, stained with ethidium bromide, and viewed on UV. The PCR product obtained had a 232-bp for the wild type and a 32-bp deletion leading to $200 \mathrm{bp}$ for mutant allele. The data obtained on CCR5 $\Delta 32$ genotypes were subjected to statistical analysis.

\section{Statistical analysis}

Alleles and genotypes were counted and their distributions between groups were determined. The Chi-square $\left(\chi^{2}\right)$ test was used to statistically compare these groups. All statistical data calculations were done with SPSS statistical package for Macintosh (release 6.1.1) (SPSS Inc., Chicago, IL). We applied survival analysis to compare the survival time after MS onset between the CCR5 genotypes. To estimate the survivor function, Kaplan-Meier product-limit was used ${ }^{34}$ and Log-rank test was used to examine the differences between two survivor curves of two CCR5 genotypes (normal vs. mutant). We also applied Cox proportional hazard model ${ }^{35}$ to test the multivariate effects including CCR5 genotypic effect, gender, and MS age of onset. These analyses were done in STATA 7.0 (Stata Corp., College Station, TX).

\section{RESULTS}

MS subjects and controls were examined for differences in allelic frequencies and genotype prevalence. No significant variation between these two groups (mutant genotype-MS = $19.7 \%$; Control $=17.1 \% ; P=0.394$ ) or with age of onset of MS ( $<35$ and $\geq 35$ years; $P=0.572$ ) was found with respect to CCR5 genotypes. However, a marked difference was observed when the Chi-square test was used to examine the potential progressive increase in certain genotypes of MS subjects across five groups with years of survival from clinical onset to death: $\leq 5$ years, $6-10$ years, $11-15$ years, $15-20$ years, and $>21$ years (see Table 1). The MS subjects with 11 genotype (normal) survived progressively more years compared to subjects with 12 and 22 genotypes (single or double mutant alleles) $(P=$ $0.00001)$.

Additionally, these groups were examined for possible differences in mutant allelic frequencies by years of survival. A significant association of the CCR5 mutant allele 2 with an early death is shown in Figure 1 with a progressive reduction in the years of survival from age at onset to the age of death with increased frequency of the CCR5 deletion allele $\left(P_{\mathrm{L}}=\right.$ 0.00005). The patients carrying the CCR 5 normal allele 1 had an earlier age at onset (mean 33.96 years) and longer survival years (mean 25.48 years) as compared to patients carrying at least one CCR5 mutant allele 2 (mean onset 35.36 years; mean survival 17.04 years).

The overall median survival time is 23 years (range 1-56) after the onset of MS. The median survival times are 24 and 16 
Table 1

Frequency of CCR5 $\Delta 32$ genotypes in MS patients in relation to years of survival (age at onset to death)

\begin{tabular}{|c|c|c|c|c|c|c|c|}
\hline \multirow[b]{3}{*}{ Years survived } & \multicolumn{6}{|c|}{ CCR5 genotypes } & \multirow{3}{*}{$\begin{array}{c}\text { Total } \\
N\end{array}$} \\
\hline & \multicolumn{2}{|c|}{11} & \multicolumn{2}{|c|}{12} & \multicolumn{2}{|c|}{22} & \\
\hline & $N$ & $\%$ & $N$ & $\%$ & $N$ & $\%$ & \\
\hline$\leq 5$ & 2 & 40.0 & 2 & 40.0 & 1 & 20.0 & 5 \\
\hline $6-10$ & 8 & 61.5 & 5 & 38.5 & & & 13 \\
\hline $11-15$ & 14 & 70.0 & 6 & 30.0 & & & 20 \\
\hline $16-20$ & 15 & 75.0 & 5 & 25.0 & & & 20 \\
\hline$<21$ & 67 & 90.5 & 7 & 9.5 & & & 74 \\
\hline Total & 106 & 80.3 & 25 & 18.9 & 1 & 0.8 & 132 \\
\hline Chi square & & & & & & & Significance \\
\hline Pearson chi-square & & & & & & & $<0.00001$ \\
\hline Linear-by linear association & & & & & & & 0.00005 \\
\hline
\end{tabular}

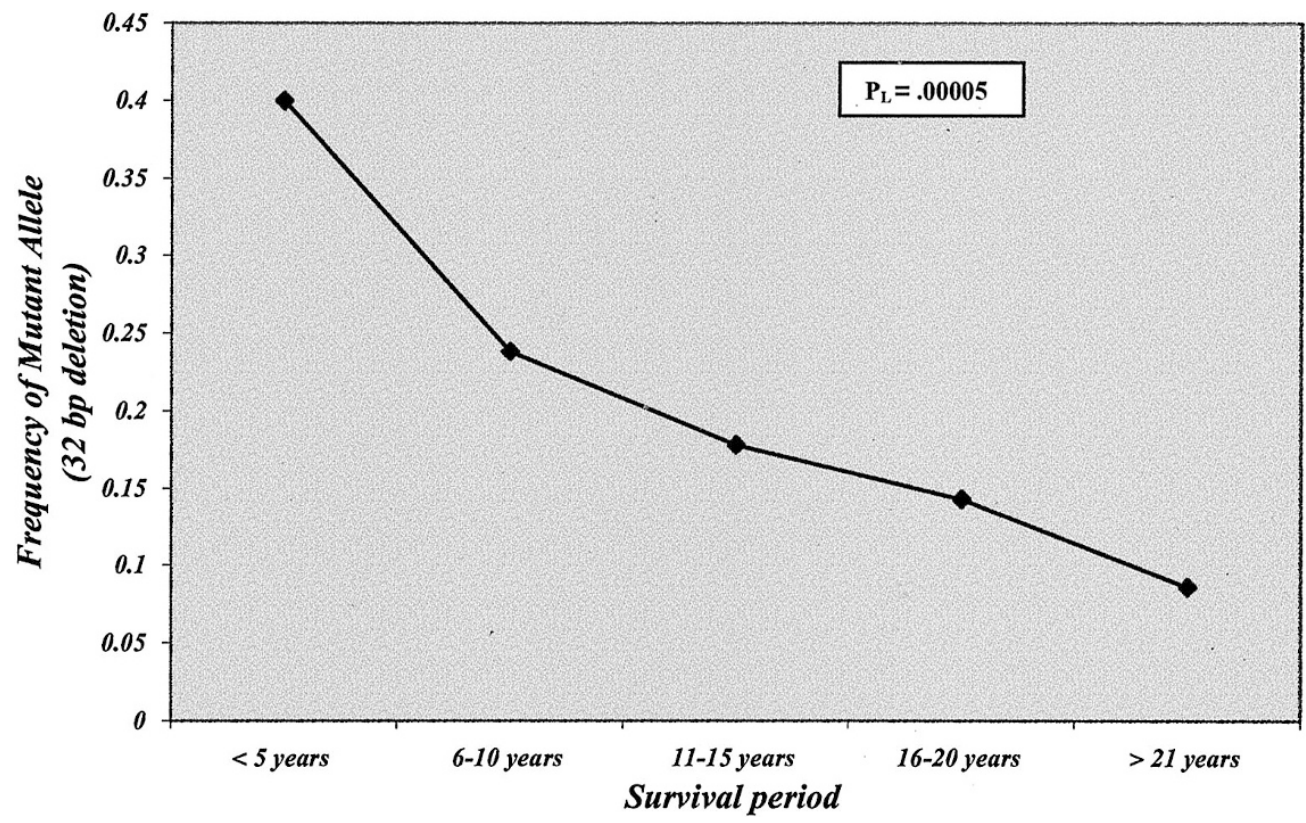

Fig. 1. Frequency of the CCR5 $\Delta 32$ mutant allele in MS patients in relation to years of survival (age of onset to death).

years and death rates are 0.039 and 0.056 for MS patients with genotypes 11 (normal) and $12+22$ (mutant), respectively. Figure 2 shows Kaplan-Meier survival curves for patients with CCR5 genotypes. The cumulative death probability is significantly higher $(P=0.00099)$ in the mutant $(12+22$ genotypes $)$ group than in normal (11 genotype) group. The cumulative death probabilities are $34 \%$ for the 11 genotype group and $69 \%$ for the $12+22$ genotype group 20 years after onset. The genotype was marginally significant with clinical subtype $(P=$ 0.033 ). Because this difference can be confounded by clinical MS subtype (mutant allele in $\mathrm{RR}=27.9 \%$ and $\mathrm{CP}=7.9 \%$ ) and the age of onset, we used the Cox regression model to test the main effect of CCR5 with simultaneous adjustment with these potential effects. The results showed that MS subtype was highly significant $(P=0.0001)$ with a greater tendency for the RR type, whereas gender and age of onset was not significant. The hazard ratio was 2.12 for $12+22$ (mutant) versus 11 (normal) genotype with $P=0.006$ (Table 2), after adjusting for MS clinical subtype (CP and RR type). This means that MS patients with CCR5 mutant genotype (12 and 22) have over twice the mortality as compared to the normal genotype, ${ }^{11}$ even after adjusting for the subtype. We have included sex as a covariate in the model and the results did not change, suggesting CCR5 and MS subtype to be the two strong covariates of survival.

Considering the high frequency of females with MS we believed that there could be a different mechanism in males versus females. Thus, we stratified our sample to separately inves- 


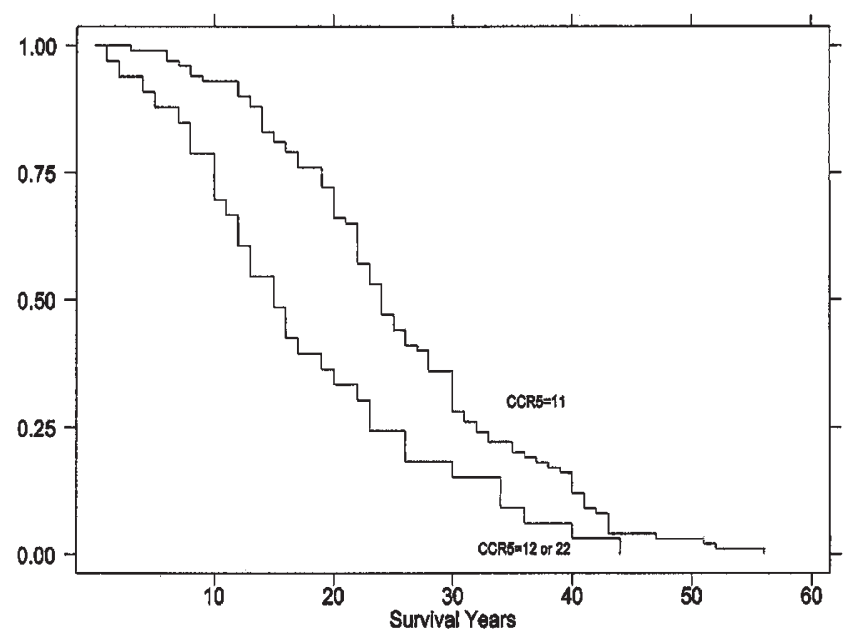

Fig. 2. Kaplan Meier product-limit survival curves for MS patients separated by CCR5 11 and 12 genotypes.

tigate this by gender. Interestingly, this genotype effect was primarily associated with female patients (Table 3$)$. The hazard ratio in females was $3.58(P=0.001 ; 95 \% \mathrm{CI}=1.74-7.38)$. There is a potential bias in as far as those patients with unknown subtypes may have worse clinical diagnosis and shorter survival years. However, this effect more likely forces our estimate downward (i.e., more conservative) if these patients were associated with CCR5 mutant genotypes.

\section{DISCUSSION}

T-cell chemokine expressions in human autoimmune disorders are believed to arise from inappropriate Th1- and Th2dominated responses. Th1 cells express CXCR3 and CCR5 and Th2 cells express CCR3 and CCR4. Chemokine receptors expressed by Th1 cells may be important as reports imply an association of MS with CNS infiltrates of CD4 cells and monocytes. To date, studies on survival of MS in relation to genotypes have not been specifically addressed and causes of death in MS cases have not been well studied. In a study of 6,068 MS cases from the Danish Multiple Sclerosis Registry, MS was noted on the death certificate as the underlying cause of death in $55.4 \%$ of cases, ${ }^{36}$ but the mechanism by which MS contributes to "premature" death has not been explicated. However, young age at onset and an initial remitting clinical course have both been shown to be significantly associated with longer survival. ${ }^{37}$ Previous studies have examined the role of the CCR5 polymorphism and MS. One study showed that the presence of a CCR5 $\Delta 32$ mutation resulted in a 3.2-year delay in the onset of familial MS, ${ }^{38}$ and the other showed that the age of disease onset was somewhat lower (about 3 years) in patients carrying the CCR $532 .{ }^{39}$ In contrast to these two studies, another report failed to demonstrate any difference. ${ }^{40}$ Although these reports have demonstrated later age at onset and increased disease intervals in CCR5 $\Delta 32$ mutants compared to wild-type alleles, these observations were not on postmortem samples and did not predict for overall survival. On the basis of the suggested links between cytokines and MS, and for the reasons given earlier, we sought to examine the possible association between CCR5 and MS.

Our study on postmortem samples failed to show an association of CCR5 deletion mutation with MS as compared to healthy controls, but showed a significant gradient in survival for MS patients when individuals having mutant CCR5 genotypes were compared to those with wild type. In diseases with multiple gene effects, genes not involved in disease susceptibility may still determine severity acting alone or could become susceptible candidates in combination with alleles of other genes. We observed that patients carrying at least one CCR5 mutant allele had an estimated 1.8 years delayed onset and 8.44 years earlier death. These observations only imply that those individuals with CCR5 mutation are predisposed to earlier mortality. An earlier report ${ }^{37}$ on association of young onset MS cases with longer survival supports our results. However, the contrast in results observed in other studies may be due in part to limitations imposed by sample size in each study, and also the reduced information on survival.

Chemokine receptor expression on T cells is related to new lesion development in MS, ${ }^{18}$ and the one possible explanation for the strong association of mutant CCR5 allele with early

Table 2

Effect of CCR 532 on survivorship in MS patients by cox proportional hazard model, with adjustment of MS subtype $(N=83)$

\begin{tabular}{lccccc}
\hline Factors & Hazard ratio (SE) & $Z$ & $P$ & Lower 95\% & Upper 95\% \\
\hline Onset age & $1.03(0.02)$ & 2.00 & 0.045 & 1.00 & 1.06 \\
CCR5 & $2.12(0.56)$ & 2.74 & 0.006 & 3.24 & 3.16 \\
MS subtype & $5.35(1.44)$ & 6.25 & 0.0001 & 9.06 \\
\hline
\end{tabular}

Table 3

Effect of CCR5 $\Delta 32$ on survivorship in female MS patients by cox proportional hazard model, with adjustment of MS subtype $(N=50)$

\begin{tabular}{lcccrr}
\hline Factors & Hazard ratio (SE) & $\mathrm{Z}$ & $P$ & Lower 95\% & Upper 95\% \\
\hline CCR5 & $3.58(1.32)$ & 3.45 & 0.001 & 1.74 & 7.38 \\
MS subtype & $19.36(10.24)$ & 5.60 & $<0.0001$ & 6.86 & 54.60 \\
\hline
\end{tabular}


death observed might be the result of enhanced CNS damage. An alternative explanation could be given based upon the CCR5 $\Delta 32$ mutation causing an up regulation of RANTES and directing the immune response away from the Th- 1 to Th-2 cells. The overall main effect of CCR5 gene observed with significantly higher cumulative death probability in mutant genotype group, in MS subtypes and in females (Table 2 and 3) suggests a complex regulation of the inflammatory response and the possible association of chemokine receptor with disease activity.

Relevant cytokine interactions are expected to vary depending on the disease process. A dysregulation of the cytokine balance can generate deleterious effects resulting in neurotoxicity, neurodegeneration, increased pathogenicity, and neurological complications. Thus genes encoding various cytokines are logical candidates to assess possible associations with MS susceptibility and the course of the disease. Our studies on postmortem samples imply that those individuals with CCR5 mutation are predisposed to earlier mortality. Thus, the presence of CCR5 mutation in patients with MS showed an exactly opposite survival effect as compared to the protective effect seen in patients with HIV-1 infection, i.e., carriers of this mutant allele were either resistant to infection by HIV-1 or had slower progression of AIDS ${ }^{41,42}$ unlike MS patients who manifested earlier death.

In polygenic disorders, genes not involved in disease susceptibility per se may play a role in determining survival. The predominance in mortality of females over males may also suggest a role for estrogenic hormones in this pathogenic milieu. In an earlier study a significant association between an X-linked gene GABRA3 and MS was observed. ${ }^{43}$ Combined, these findings are consistent with the theory that generation of clinical manifestations involves both cytokine action and cytokine modulation of neurotransmitters. Understanding the role of the CCR5 gene and characterization of cytokine-cytokine interaction and cytokine-neurotransmitter interactions is important. The clinical relevance of this CCR5 mutation in patients with MS could help in designing a therapeutic regimen and may serve as a prognostic marker.

\section{ACKNOWLEDGMENTS}

This work was supported by the Peg Liddle Foundation at Eisenhower Medical Center, Rancho Mirage, CA. Study material support from Department of Medical Genetics, City of Hope Medical Center, Duarte, CA and Neurology \& Research Services, VA, West Los Angeles Health care Center \& Dept. of Neurology, UCLA, Los Angeles, CA. The authors thank M. Berkeley, G. Espinoza, C. Mendes, and A. Moreno for their expert assistance in the study.

\section{References}

1. Karpus, W, J Lukacs NW, McRae BL, Strieter RM, Kunkel SL, Miller SD. An important role for the chemokine macrophage inflammatory protein-1 alpha in the pathogenesis of the T cell-mediated autoimmune disease, experimental autoimmune encephalomyelitis. J Immunol 1995;155:5003-5010.

2. Ransohoff RM. Mechanisms of inflammation in MS tissue: adhesion molecules and chemokines. J Neuroimmunol 1999;98:57-68.
3. Huang Y, Paxton WA, Wolinsky SM, Neumann A, Zhang L, He T et al. The role of a mutant CCR5 allele in HIV-1 transmission and disease progression. Nat Med 1996;2:1240-1243.

4. Biti R, French R, Young J, Bennetts B, Stewart G. HIV infection in an individual homozygous for the CCR5 deletion allele. Nat Med 1997;3:252-253.

5. O'Brien TR, Winkler C, Dean M, Nelson JAE, Carrington M, Michael NL. HIV-1 infection in an homozygous for CCR5 delta 32. Lancet 1997;349:1219-1220.

6. Mummidi S, Ahuja S, Gonzalez E, Anderson SA, Santiago EN, Stephan KT et al. Genealogy of the CCR5 locus and chemokine system gene variants associated with altered rates of HIV-1 disease progression. Nature Med 1998;4:786-793.

7. de Roda Husman AM, Blaak H, Brouwer M, Schuitemaker H. CC chemokine receptor 5 cell-surface expression in relation to CC chemokine receptor 5 genotype and the clinical course of HIV-1 infection. J Immunol 1999;163:4597-4603.

8. Daar ES, Lynn H, Donfield S, Gomperts E, Hilgartner MW, Hoots K et al. Effects of plasma HIV RNA, CD4+ lymphocytes and the chemokine receptors CCR5 and CCR2b on HIV disease progression in hemophiliacs. JAIDS 1999;21:317-325.

9. Cone L. A, Espinoza G, Moreno A, Downs M. CC-CKR5 (CCR5) delta 32 alters the clinical course and immune status of HIV infection [abstract]. 13th Int. AIDS conference, Durban, South Africa, July 8-13, 2000;TuPeA 3107:320.

10. Balashov KE, Rottman JB, Weiner HL, Hancock WW. CCR5 (+) and CXCR3 (+) T cells are increased in multiple sclerosis and their ligands MIP-1 alpha and IP-10 are expressed in demyelinating brain lesions. Proc Natl Acad Sci U S A 1999;96:6873-6878.

11. Sorensen TL, Tani M, Jensen J, Pierce V, Lucchinetti C, Folcik VA et al. Expression of specific chemokines and chemokine receptors in the central nervous system of multiple sclerosis patients. J Clin Invest 1999;103:807-15.

12. Simpson J, Rezaie P, Newcombe J, Cuzner ML, Male D, Woodroofe MN. Expression of the beta-chemokine receptors CCR2, CCR3 and CCR5 in multiple sclerosis central nervous system tissue. J Neuroimmunol 2000;108:192-200.

13. Zhang GX, Baker CM, Kolson DL, Rostomi AM. Chemokines and chemokine receptors in the pathogenesis of multiple sclerosis. Mult Scler 2000;6:3-13.

14. Strunk T, Bubel S, Mascher B, Schlenke P, Kirchner H, Wandinger KP. Increased numbers of CCR5 + interferon- $\gamma$ - and tumor necrosis factor- $\alpha$-secreting T lymphocytes in multiple sclerosis patients. Ann Neurol 2000;47:269-273.

15. Challoner PB, Smith KT, Parker JD, MacLeod DL, Coulter SN, Rose TM et al. Plaque- associated expression of human herpesvirus 6 in multiple sclerosis. Proc Natl Acad Sci U S A 1995;92:7440-7444.

16. Sanders VJ, Felisan S, Waddell A, Tourtellotte WW. Detection of herpesviridae in postmortem multiple sclerosis brain tissue and controls by polymerase chain reaction. J Neurovirol 1996, Aug; 2: 249-258.

17. Knox KK, Brewer JH, Henry JM, Harington DJ, Carrigan DR. Human herpes virus 6 and multiple sclerosis. Systemic active infections in patients with early disease. Clin Infect Dis 2000;31:894-903.

18. Eikelenboom MJ, Killestein J, Izeboud T, Lalkers NF, van Lier RA, BarKhof F et al. Chemokine receptor expression on $\mathrm{T}$ cells is related to new lesion development in multiple sclerosis. J Neuroimmunol 2002;133:225-232.

19. Bonecchi R, Bianchi G, Bordignon PP, D'Ambrosio D, Lang R, Borsatti A et al. Differential expression of chemokine receptors and chemotactic responsiveness of type1 T helper cells (TH-1s) and TH-2s. J Exp Med 1998;187:129-134.

20. Paxton WA, Neumann AU, Kang S, Deutch L, Brown RC, Koup RA et al. RANTES production from $\mathrm{CD} 4+$ lymphocytes correlates with host genotype and rates of human immuno-deficiency virus type 1 disease progression. J Infect Dis 2001;183: $1678-1681$.

21. Baranzini SE, Elfstrom C, Chang SY, Butunoi C, Murray R, Higuchi R et al. Transcriptional analysis of multiple sclerosis brain lesions reveals a complex pattern of cytokine expression. J Immunol 2000;165:6576-6582.

22. Ebers GC, Sadovnick AD. The role of genetic factors in multiple sclerosis susceptibility. J Neuroimmunol 1994;54:1-17.

23. Oksenberg JR, Seboun E, Hauser SL. Genetics of demyelinating diseases. Brain Pathol 1996;6:289-302.

24. Sawcer S, Jones HB, Feakes R, Gray J, Smaldon N, Chataway J et al. A genome screen in multiple sclerosis reveals susceptibility loci on chromosome 6p21 and 17q22. Nat Genet 1996;13:464-468.

25. Hooper-van Veen T, Schrjver HM, Zwiers A, Crusius JB, Knol DL, Kalkers NF et al. The interleukin-1 gene family in multiple sclerosis susceptibility and disease course. Multi Scler 2003;9:535-539.

26. Teutsch SM, Booth DR, Bennetts BH, Heard RN, Stewart GJ. Identification of 11 novel and common single nucleotide polymorphisms in the interleukin-7 receptoralpha gene and their association with multiple sclerosis. Eur J Hum Genet 2003;11: $509-515$.

27. Masterman T, Ligers A, Zhang Z, Heilgren D, Salter H, Anvret M et al. CTLA4 dimorphisms and the multiple sclerosis phenotype. J Neuroimmunology 2002;131: $208-212$.

28. Schreiber K, Otura AB, Ryder LP, Madsen HO, Jorgensen OS, Sverjgaard A et al. Disease severity in Danish multiple sclerosis patients evaluated by MRI and three 
genetic markers (HLA-DRB1*1501, CCR5 deletion mutation, apolipoprotein E). Mult Scler 2002;8:295-298.

29. Poser CM. The dissemination of multiple sclerosis: a Viking saga? A historical assay. Ann Neurol 1994;36:S231-S243.

30. Rosati G. The prevalence of Multiple sclerosis in the world: an update. Neurol Sci, Apr 2001;22:117-139.

31. Martinson JJ, Chapman NH, Rees DC, Liu Y. T, Clegg JB. Global distribution of the CCR5 gene 32 base pair deletion. Nat Genet 1997;16:100-102.

32. Lucotte G, Mercier G. Distribution of the CCR5 gene 32-bp deletion in Europe. J Acquire Immune Def Syn Hum Retro Virol 1998;19:174-177.

33. Siao CJ, Tsirka SE. Extracellular proteases and neuronal cell death. Cell Mol Biol (Noisy-le-grand) 2002;48:151-161.

34. Kaplan EL, Meier P. Nonparametric estimation from incomplete observations. JAm Stat Assoc 1958;53:457-481.

35. Cox DR, Oaks D. Analysis of survival data. London UK: Chapman \& Hall, 1984

36. Koch-Henriksen N, Bronnum-Hansen H, Stenager E. Underlying cause of death in Danish patients with multiple sclerosis: results from the Danish Multiple Sclerosis Registry. J Neurol Neurosurg Psychiatry 1998;65:56-59.

37. Midgard R, Albrektsen G, Riise T, Kvale G, Nyland H. Prognostic factors for survival in multiple sclerosis: a longitudinal, population based study in More and Romsdal, Norway. J Neurol Neurosurg Psychiatry 1995;58:417-421.
38. Barcellos LF, Schito AM, Rimmler JB, Vittinghoff E, Shih A, Lincoln R et al. CCchemokine receptor 5 polymorphism and age at onset in familial sclerosis. Immunogenet 2000;51:281-288.

39. Sellebjerg F, Madsen HO, Jensen CV, Jensen J, Garred P. CCR5 delta 32, matrix metallo- proteinase 9 and disease activity in multiple sclerosis. J Neuroimmunol 2000;102:98-106.

40. Bennetts BH, Teutsch SM, Buhler MM, Heard RNS, Stewart GJ. The CCR5 deletion mutation fails to protect against multiple sclerosis. Human Immuno 1997;58:52-59.

41. Dean M, Carrington M, Winkler C, Huttley GA, Smith MW, Allikmets R et al. Genetic restriction of HIV-1 infection and progression of AIDS by a deletion allele of the CCR5 structural gene. Hemophilia growth and Development study. Multicenter AIDS Cohort study, Multicenter Hemophilia study, San Francisco Cohort study, Alive study. Science 1996;273:1856-1862.

42. Wu L, Paxton WA, Kassam N, Ruffing N, Rottman JB, Sullivan N. CCR levels and expression pattern correlate with the infectability by macrophage-tropic HIV-1, in vitro. J Exp Med 1997;185:1681-1691.

43. Gade-Andavolu R, MacMurray J, Blake H, Muhleman D, Tourtellotte W, Coming DE. Association between $\gamma$-aminobutyric acid A3 receptor gene and Multiple Sclerosis. Arch Neurol 1998;55:513-516. 